\title{
Variaciones en las conductas no coitales de los chicos
}

\author{
Variations of noncoital behavior of males \\ Encarnación Sueiro, Antonio López-Castedo, María Isabel Nóvoa \\ Fac. CC. de la Educación. (U.de Vigo-Campus de Ourense)
}

\section{Resumen}

En esta investigación se analizan los cambios ocurridos en las últimas conductas no coitales, en los hombres, durante un intervalo de 10 años. Para ello se llevo a cabo un estudio longitudinal, diacrónico o transversal y de tendencia sobre una muestra de 2245 chicos universitarios (curso 97-98) y 856 (07-08), que realizaban el primer y último curso en la Universidad de Vigo. Se les aplicó una encuesta estructurada, anónima y voluntaria en la que se recogía la edad y variables sexuales, referidas a las conductas no coitales. La edad media de las hombres universitarios, del curso 97-98, es de 20.85 años y la del curso 07-08, de 21.42 años. Los datos se analizaron mediante el programa estadístico SPSS 21.0. Hay diferencias significativas $(\mathrm{p}<.05)$ entre ambos grupos en: porcentaje de autoestimulación, frecuencia y edad de inicio, existencia de actividad sexual con otra persona, frecuencia de diferentes conductas sexuales no coitales y coitales y su edad de inicio.

Palabras clave: cambios, conductas no coitales, universitarios.

\section{Abstract}

In the last decades, sexuality is one of the facets that raises more interest among evolutionary researchers. In this research the change occurred in the noncoital behavior that are analyzed in males, for a period of 10 years. It took a very lengthy study, diachronic o transversal, with a trend over a sample of 2245 young university students (course 97-98) and 856 (course 07-08), in they were in their first and last year of their university course, in the University of Vigo. It was applied a structured survey, anonymous and voluntary, in which was the ages and variables were gathered, contemplating their noncoital behavior. The average age of university male, course $97-98$, was of the ages 20 85 years old and the course $07-08$, was of the ages 21-42 years old. The data was anaylzed using the statistical program SPSS 21.0.

Keywords: changes, noncoital, universitary males.

\section{Objetivo}

Saber los cambios ocurridos en as conductas sexuales no coitales realizadas por los jóvenes.

\section{Método}

Es un estudio longitudinal, diacrónico o transversal y de tendencia, realizado con una muestra de 2245 y 856 jóvenes universitarios que realizaban el primer y último curso, en el Campus de Vigo, de Ourense y de Pontevedra, de la Universidad Sur de Galicia, en los cursos 1997-1998 y 2997-2008, respectivamente. A toda se le aplicó una encuesta estructurada, anónima y voluntaria en la que se recogía la edad y variables referidas a las primeras conductas sexuales no coitales: existencia de autoestimulación, edad de inicio y frecuencia, actividad sexual realizada con otra persona diferente del coito, diferentes conductas sexuales ejecutadas (cita, beso, beso profundo, estimulación de los senos por encima de la ropa, estimulación de los senos debajo de la ropa, estimulación genital activa, estimulación genital pasiva, contacto intergenital, coito vaginal/anal con una o varias parejas) y su edad de comienzo.al primer coito: su inicio y edad de comienzo, sexo de la primera pareja, tipo de pareja, sentimientos en ese primer encuentro, tiempo de conocimiento previo, razones que la motivaron, lugar en el que aconteció, frecuencia y número de parejas durante el primer año, existencia de orgasmo, consumo etílico, uso de protección, tipo, existencia de problemas con su uso y quien la propone. Los datos se analizan mediante el programa estadístico SPSS 17.0.

\section{Resultados}

El $94.8 \%$ de los hombres del curso $97-98$ y el $96.8 \%$ de los varones del curso 08-09 manifestaron que se han autoestimulado; mientras que, un $4.0 \%$ de la primera muestra y un $1.3 \%$ de la segunda, declararon que no se habían masturbado, encontrando diferencias significativas entre ambos grupos de varones $\left(\chi^{2}=\right.$ $66.21 ; \mathrm{p}=0.000)$

El porcentaje más elevado (35.8\%), que corresponde a los hombres del curso 97-98, manifiestaron haberse masturbado de 1 a 3 veces a la semana, porcentajes que se incrementa a un $37.0 \%$ en los hombres del curso 07 08. Cabe destacar que un $22.3 \%$ de la primera muestra y un $27.2 \%$ de la segunda, se autoestimularon 4 ó más veces por semana, mientras que el $27.3 \%$ y el $23.4 \%$, respectivamente, realizaron esta práctica de 1 a 3 veces al mes. El análisis estadístico nos muestra que existen diferencias significativas $\left(\chi^{2}=20.39 ; p=0.002\right)$ entre ambos cursos, en los hombres.

Se pone de manifiesto que el porcentaje más elevado, corresponde al intervalo de edad de 13 a 16 años, siendo 
para las hombres del curso $97-98$ de un $57.9 \%$, y decreciendo a un $53.1 \%$ para los del curso 07-08. Es de resaltar que el $31.2 \%$ de los varones del curso $97-98$ y el $39.4 \%$ de los varones del curso 07-08 manifiestan haber comenzado entre los 9-12 años la masturbación. Los restantes intervalos de edad hacen referencia a pequeños porcentajes de ambos grupos de hombres y aparecen bastantes igualados. En el análisis estadístico se observan diferencias significativas entre ambos grupos $\left(\chi^{2}=30.48 ; \mathrm{p}=0.000\right)$.

Se percibe que el $79.3 \%$ de los hombres del curso 9798, y el $91.0 \%$, de los del curso 07-08, afirmaron haber tenido alguna actividad sexual con otra persona; mientras un $16.7 \%$ de la primera muestra y un $6.1 \%$ de la segunda aseguraron no haber realizado actividad sexual alguna con otra persona, encontrando diferencias significativas entre los hombres en función del curso analizado $\left(\chi^{2}=66.21 ; \mathrm{p}=0.000\right)$.

El porcentaje de hombres del curso 97-98 que tuvieron cita es de $75.3 \%$ que se incrementa en el varón del curso $07-08$, a un $84.9 \%$. A su vez, se aprecia un aumento en las conductas sexuales del curso 07-08: beso (de un $78.0 \%$ pasa a un $88.4 \%$ ), beso profundo (de un $77.6 \%$ se incrementa a un $85.7 \%$ ), estimulación de los senos encima de la ropa (de un $69.5 \%$ asciende a un $81.4 \%$ ), estimulación de los senos debajo de la ropa (de un $68.2 \%$ aumenta a un $81.9 \%$ ), estimulación genital activa (de un $60.0 \%$ se eleva a un $76.6 \%$ ), estimulación genital pasiva (de un $56.7 \%$ asciende a un $75.2 \%$ ), contacto intergenital (de un $54.1 \%$ pasa a un $73.9 \%$ ) y coito vaginal/anal con varias parejas (de un $6.2 \%$ se eleva a $18.5 \%$ ). Cabe subrayar los datos referidos al coito vaginal/anal con una persona, que pasa de un $50.6 \%$ en el curso $97-98$, a un $78.3 \%$ al curso $07-08$. El análisis estadístico pone de manifiesto que existen diferencias significativas en todas las conductas sexuales entre ambos grupos de varones: cita $\left(\chi^{2}=\right.$ $31.39 ; \mathrm{p}=0.000)$, beso $\left(\chi^{2}=43.14 ; \mathrm{p}=0.000\right)$, beso profundo $\left(\chi^{2}=28.33 ; \mathrm{p}=0.000\right)$, estimulación de los senos encima de la ropa $\left(\chi^{2}=46.81 ; \mathrm{p}=0.000\right)$, estimulación de los senos debajo de la ropa $\left(\chi^{2}=63.61\right.$; $\mathrm{p}=0.000)$, estimulación genital activa $\left(\chi^{2}=83.17 ; \mathrm{p}=\right.$ $0.000)$, estimulación genital pasiva $\left(\chi^{2}=97.12 ; \mathrm{p}=\right.$ $0.000)$, contacto intergenital $(113.27 ; \mathrm{p}=0.000)$, coito vaginal/anal con una persona $\left(\chi^{2}=193.26 ; p=0.000\right)$ y coito vaginal/anal con varias personas $\left(\chi^{2}=111.09 ; \mathrm{p}=\right.$ 0.000).

La edad de inicio de la cita es de 14.36 años en el caso de los hombres del curso 97-98 y de 13.72 años en los hombres del curso 07-08, sin embargo todas las edades decrecen respecto al curso 07-08 en las siguientes conductas sexuales: beso (de 14.32 años a 13.26 años), beso profundo (de 15.03 años a 13.99 años), estimulación senos encima de la ropa (de 15.79 años a 14.99 años), estimulación senos debajo de la ropa (de 16.14 años a 15.41 años), estimulación genital activa (de 16.87 años a 16.08 años), estimulación genital pasiva (de 16.97 años a 16.11 años), contacto intergenital (de 17.54 años a 16.52 años) y coito anal/vaginal con varias parejas (de 18.05 años a 17.44 años). Por otra parte, subrayar la edad de inicio del coito del varón del curso 97-98 (18.05 años) respecto al varón del curso 07-08 (16.97 años). Se aprecian diferencias significativas entre los hombres de ambas muestras en las siguientes conductas sexuales: estimulación de los senos encima $(\mathrm{t}=7.033 \mathrm{p}=0.008)$ y por debajo de la ropa $(\mathrm{t}=11.75 ; \mathrm{p}=0.001)$, estimulación genital activa $(\mathrm{t}=17.42 ; \mathrm{p}=0.000)$ y pasiva $(\mathrm{t}=18.97 ; \mathrm{p}=0.001)$, contacto intergenital $(\mathrm{t}=22.36 ; \mathrm{p}=0.000)$ y coito vaginal/anal con una pareja $(\mathrm{t}=18.61 ; \mathrm{p}=0.000)$; mientras que no se encuentran estas diferencias en la cita $(\mathrm{t}=0.13 ; \mathrm{p}=0.714)$, beso $(\mathrm{t}=1.84 ; \mathrm{p}=0.175)$, beso profundo $(\mathrm{t}=3.71 ; \mathrm{p}=0.054)$ y coito vaginal/anal con varias parejas $(\mathrm{t}=0.30 ; \mathrm{p}=0.584)$.

\section{Discusión}

En nuestros resultados encontramos casi que la totalidad de los hombres del curso 1997-98 y del curso 2007-08 (94.8\% y 96.8\%, respectivamente), se han masturbado, destacando que tras diez años de evolución se observa que el varón, en estos momentos, se autoestimula en mayor medida que hace 10 años.

En la investigación de Doval et al. (1987), se señala que un porcentaje algo superior al encontrado en este estudio, un $97 \%$ de los varones, se han masturbado; sin embargo, en un trabajo posterior (Doval et al., 1989), revela un porcentaje inferior $74.0 \%$. Mesa et al. (2004), afirman que el $100.0 \%$ se habían masturbado, porcentaje ligeramente superior al encontrado con los varones del curso 2007-08; y, finalmente, Lasheras et al. (2005); ponen de manifiesto que el $99 \%$ de los varones se masturbaron alguna vez, resultado que coincide con el encontrado en la muestra del curso 2007-08.

Las investigaciones realizadas con niños de 9 a 14 años, indican que la masturbación ya se da en el $8.3 \%$ entre los 9-10 años y su prevalencia llega a ser del $87.3 \%$ a los 13-14 años (Ballester y Gil, 2006).

Por lo que hace referencia a la frecuencia de la autoestimulación, durante el primer año en que iniciaron esta práctica sexual, los porcentajes más altos se encuentran en el intervalo de 1 a 3 veces a la semana, (35.8\%, para el curso $1997-98$, y $37.0 \%$, para el 200708 ) y 4 o más veces $(22.3 \%$ y $27.2 \%$, respectivamente).

Las distintas investigaciones ponen de manifiesto que un $29.6 \%$ de los varones practican la autoestimulación de 1 a 3 veces a la semana (Sueiro y Diéguez, 2001); que la frecuencia de la masturbación en los varones (46\%) es mayoritariamente de 1 a 3 veces a la semana, un $34 \%$ la frecuencia es de 1 a 3 veces al mes, para un $16 \%$ más de 5 veces por semana y para $1 \%$ menos de 1 a 3 veces al mes (Lasheras, et al., 2005); y, datos semejantes se encuentran en la población no universitaria (Diz et al., 2003), donde los porcentajes 
mayoritarios para el hombre se sitúan en el intervalo de 1 a 3 veces a la semana.

Por otra parte, las investigaciones realizadas con niños de 9 a 14 años, se observa que en el intervalo de 11-12 años, el mayor porcentaje (54\%) se masturba entre 1 y 3 veces a la semana, mientras que en el intervalo de 13-14 años la mayoría (79\%) se masturba entre todos los días y 2-3 veces a la semana (Ballester y Gil, 2006).

Los diferentes estudios ratifican nuestros resultados, significando que la masturbación es una conducta que se da con frecuencia en los hombres jóvenes, y una tendencia que a lo largo del tiempo se practica en mayor medida.

Con respecto a la edad de inicio de la primera masturbación, los porcentajes más altos se encuentran en la franja de edad de 13 a 16 años en las dos muestras (curso 1997-98 y 2007-08), siendo los porcentajes de $57.9 \%$ y $53.1 \%$, respectivamente, es de señalar, la franja de edad de 9 a 12 años, que aglutina un $31.2 \%$ de los varones de la primera muestra y se incrementa a un $39.4 \%$ para los varones de la segunda muestra. Así, en los comienzos de la década de los 70, alrededor del $50 \%$ de los chicos se habían masturbado hacia la edad de los 15 años; mientras que, a finales de la misma década, los porcentajes del 70\% para los chicos (Dreyer, 1982). Teniendo presente los datos de esta investigación, podemos afirmar que este incremento se sigue dando, ya que para un $93.3 \%$ del alumnado del curso 1997-98 a los 16 años ya se habían masturbado por primera vez, incrementándose a un $96.8 \%$ diez años después.

Diferentes estudios presentan resultados que están en la misma línea de esta investigación. Así, en la investigación de Doval et al. (1987), se indica que el $34.28 \%$ de los chicos la inició a los 11 años o antes y el $59.04 \%$ de los que se autoestimulaban, a los 12 años o más. Lasheras et al. (2005), afirman que la masturbación se inició a los 13-14 años en los chicos.

Cuando se estudia el hecho de haber tenido alguna actividad sexual con otra persona, se encuentra que el $79.3 \%$ de los varones, del curso 1997-98, manifestaron haberla tenido, esto se incrementa para los jóvenes del curso 2007-08, a un 91.0\%. En este sentido, García et al. (1995), observan que un $11.6 \%$ de los varones han tenido poco o ningún contacto con el sexo opuesto y un $15.9 \%$ de los chicos tienen una experiencia limitada de actividades sexuales (besos y caricias por encima de la ropa), porcentaje que viene a corroborar los encontrados en el curso 1997-98. En el estudio de Gascón et al. (2003), se señala que un $19.3 \%$ de los varones no tuvieron ningún tipo de actividad sexual. Por su parte, en la Encuesta de Salud y Hábitos Sexuales (2003), revela que un $89.4 \%$ de los varones declaran haber tenido relaciones sexuales (completas e incompletas); sin embargo, en la $2^{a}$ Encuesta Schering de Sexualidad y Anticoncepción en la Juventud Española (2005), realizada entre jóvenes con edades comprendidas entre 15 y 24 años, se pone de manifiesto que el $92.7 \%$ de los chicos, han mantenido relaciones sexuales -completas o incompletas- en alguna ocasión, resultados estos dos últimos que concuerdan totalmente con los de la muestra del curso 2007-08. Otros estudios con población no española, que analizan la conducta sexual de estudiantes varones, entre los 15 y 19 años, afirman que el 90\% participó en algún tipo de actividad sexual a los 18 años (Kapamadzija et al., 2000).

Si nos centramos en las prácticas sexuales realizadas, hay un incremento en el número de varones del curso 2007-08, respecto a los del curso 1997-98, en todas las prácticas analizadas (beso, beso profundo, estimulación senos, estimulación genital, contacto intergenital y coito). Cabe destacar el coito vaginal/anal con una persona, donde el porcentaje pasa del curso 1997-98 al 2007-08, de un 50.6\% a un 78.3\%. Estos datos vienen a corroborar la investigación de Sueiro y Diéguez (2001), sobre varones universitarios, evidenciando que a medida que nos aproximamos al coito, la presencia de los comportamientos sexuales tienden a disminuir. Este porcentaje desciende del $78.3 \%$ para el beso a un $76.5 \%$ para el beso profundo, en la estimulación de los senos encima de la ropa y por debajo de la ropa $(64.1 \%$ y $63.1 \%$, respectivamente), para la estimulación genital activa y pasiva (53.0\% y $51.6 \%$, respectivamente), en el contacto intergenital (49.3\%) hasta llegar al $48.4 \%$ para el coito vaginal/anal con una pareja y al $9.2 \%$ para el coito vaginal/anal con varias parejas.

En diferentes estudios observamos cómo el porcentaje de universitarios que realiza el coito se incrementa paulatinamente según pasan los años. Así, García et al. (1995), indican que un $51.1 \%$ de los varones tuvo relaciones coitales; Rodríguez y Traverso (2007), el $84.4 \%$ de los varones mantuvieron relaciones coitales. En síntesis, todos estos estudios corroboran los datos obtenidos, y nos permiten afirmar que en los últimos 10 años el porcentaje de hombres que mantienen relaciones coitales es superior al de la década anterior.

Los resultados obtenidos en los estudios del Instituto de la Juventud, también confirma el incremento progresivo de los porcentajes de varones que tienen relaciones coitales, en una misma franja de edad con respecto a la experiencia sexual completa: IJE-96, muestra que el $60 \%$ de los varones comprendidos entre 15 y 29 años han tenido relaciones sexuales completas, aunque estos porcentajes son diferentes en función de los diversos tramos de edad (los jóvenes de 18 a 20 años sería un 50\% y para el intervalo de 21 a 24 años sería un $74 \%$ ); en el IJE-2000, representan un $62 \%$ de los varones entre 15 y 29 años (para tramos de edad de 18 a 20 años sería un 50\% y para el intervalo de 21 a 24 años de $76 \%$ ); IJE 2004, el $82 \%$ de los chicos entre 15 y 29 años tuvieron coito (para el tramo de edad de 18 a 20 años es de un $77.7 \%$, y de 21 a 24 años de un 92.2\%); y en el IJE 2008 , el $83.8 \%$ de los chicos entre 15 y 29 años $(85.9 \%$ de los hombres de 18 a 20 años y de un $93.0 \%$ de los hombres de 21 a 24 años). 
Los datos obtenidos por el IJE-96 y por el IJE-08 ratifican los obtenidos para el curso 1997-98 y 2007-08, lo que nos pone de manifiesto que los varones actuales realizan más prácticas sexuales que los de la década anterior. Por otra parte, en la $2^{\mathrm{a}}$ Encuesta Schering de Sexualidad y Anticoncepción en la Juventud Española (2005), se refiere que el $75.8 \%$ de los chicos, han mantenido relaciones sexuales con penetración, resultados estos que concuerdan con la Encuesta de Salud y Hábitos Sexuales del INE (2003)y por los encontrados en esta investigación con el alumnado del curso 1997-98. A su vez, Failde et al. (2008), mencionan que el $77.1 \%$ de los hombres han practicado coito vaginal, resultado que está en la misma línea que el obtenido con los hombres del curso 2007-08.

Cuando se analizan los estudios de alumnos de enseñanza no universitaria, debido a su menor edad, los porcentajes decrecen: Oraá (1996), observa que un 34\% de los hombres han practicado el coito; Navarro et al (2006), indican que el $19 \%$ de las varones tuvieron coito; Diz, et al. (2003), refieren que el $50.7 \%$ de los hombres tuvo relaciones coitales; Lameiras et al (2004b), afirman que un $36.6 \%$ de los chicos son sexualmente activos; Mesa et al. (2004), destacan que el $30.1 \%$ tuvieron su primer coito; Teva et al. (2009b), manifiestan que el $26.7 \%$ tenían experiencia sexual coital.

A la hora de evaluar la variable referida a la edad de inicio de las diferentes prácticas sexuales realizadas, encontramos un descenso en la misma; los varones del curso 2007-08 comenzaron a edades más tempranas que los varones del curso 1997-98, observando que en algunas de las prácticas estudiadas este descenso es significativo (estimulación de los senos encima y debajo de la ropa, estimulación genital activa y pasiva, contacto intergenital y coito vaginal/anal con una pareja). Por lo que hace referencia al coito, la edad media descendió desde 18.0 años para los varones del curso 1997-98 a 17.4 años para los varones del curso 2007-08. Si analizamos en un primer momento las prácticas no coitales, las primeras prácticas a las que se accede son los besos, en el curso 1997-98 a los 14.32 años y en el curso 2007-08 descienden a un 13.2 años. Oraá (1996), indica que las primeras conductas sexuales que se acceden son los besos aproximadamente a los 14 años, estos resultados son coincidentes con los de la muestra del curso 1996-97; Sueiro y Diéguez (2001), apuntan que el hombre a la edad de 14.3 años tuvo su primera cita, a los 14.2 años el beso, a los 14.8 años beso profundo, a los 16.0 años estimulación de los senos encima de la ropa, a los 15.9 años estimulación de los senos debajo de la ropa, a los 17.0 años estimulación genital activa o pasiva, a los 17.5 años contacto intergenital, corroborando los datos obtenidos con la muestra del curso 1997-98; Diz et al. (2003), refieren que la edad media de la cita para el hombre es 12.9 años y la del coito de 16.6, utilizando una muestra más joven y procedente del medio rural; Mesa et al. (2004), anota que $46.8 \%$ de los varones realizaron su primer beso a los 14-16 años y el $38.6 \%$ lo ha alcanzado antes de los 13 años.

Si nos centramos en la conducta coital, un análisis pormenorizado de las mismas, muestra que globalmente los hombres han ido descendiendo la edad de inicio de las relaciones coitales. En las investigaciones con población universitaria, García et al (1995), afirman que el grupo más numeroso de hombres ha tenido su primera relación sexual entre los 14 y 22 años, y en el intervalo de 18 y 19 años; Rodríguez y Traverso (2007), sitúan la edad de inicio de los varones en 17.7 años, edad superior a la muestra del curso 2007-08.

Si nos centramos en estudios con población juvenil o adulta, en el IJE-96, la edad promedio en la que se realiza la iniciación sexual en los hombres es de 17 años y 7 meses; IJE-2000, de 17 años y 4 meses; IJE del 2004, es de 17 años y 4 meses; y en el IJE-2008, se sitúa en 16 años y 6 meses. Si se elimina en el IJE-2008, el grupo de edad de 15 a 17 años, la media obtenida aumenta para los varones en casi medio año y se sitúa en un intermedio de 17 años para los varones. Los resultados del IJE-96 e IJE-08, corroboran los de este estudio.

Por otra parte, la encuesta del INE-2003, para la edad 18/29 años, sitúa la edad de inicio de los coitos en 17.5 años para los varones, siendo coincidentes con las obtenidas en esta investigación, teniendo en cuenta que las muestras, una es anterior y otra posterior, y la tendencia es ir disminuyendo la edad de inicio de las relaciones coitales según el incremento de los años. Lo mismo nos acontece con el estudio de Ramírez et al. (2002), sobre métodos anticonceptivos en la población Andaluza, y en una población con una edad comprendida entre los 15 y 49 años, la edad media de inicio de las relaciones coitales en el hombre es de 17.3 años. A su vez, los resultados de la $2^{\text {a }}$ Encuesta Schering de Sexualidad y Anticoncepción en la Juventud Española (2005), coinciden con los de la muestra del curso 2007-08, al indicar que la edad media de los chicos es de 16.5 años, pero para los varones entre $20 \mathrm{y}$ 24 años las edades se incrementan a un 16.8 años concordando con las encontradas en esta investigación.

$\mathrm{Si}$ analizamos estudios de población no universitaria, observamos en la investigación de Oraá (1996), que la edad media para el primer coito fue a los 17 años; Hidalgo et al. (2000), la sitúan en 15.4 años; Diz et al. (2003), refieren una edad media de 16.6 años; Moreno Rodríguez et al. (2004), la ubican en los 15.3 años; y Teva et al. (2009b), manifiestan una edad de inicio del primer coito para los hombres adolescentes en los 14.8 años. En estos resultados se observa que las edades son más bajas que los datos encontrados con las muestras de la investigación, debido a la utilización de muestras de edades inferiores. 


\section{Referencias}

Ballester, R. y Gil, M.D. (2006). La sexualidad en niños de 9 a 14 años. Psicothema, 18 (1), 25-30.

Diz, M.C., Sueiro, E., Chas, M.D., y Diéguez, J.L. (2003). Comportamientos sexuales de adolescentes del medio rural gallego ( $3^{\mathrm{a}}$ Parte). Cuadernos de Medicina Psicosomatica y Psiquiatría de Enlace, (67/68), 46-60.

Doval, J.L., Perdiz, C., Seare, M. M., Sueiro, E., y Valencia, L. (1987). La sexualidad en adolescentes en Ourense. Actas III Congreso Estatal de Planificación Familiar. Valladolid, 59.

Doval, J.L., Perdiz, C., y Sueiro, E. (1989). Programa de anticoncepción y sexualidad para adolescentes. Revista Sexpol. Boletín de información sexológica, (14), 27-31.

Dreyer, P.H. (1982). Sexuality during adolescence. En B.B.Wolman (Ed.), Handbook of developmental psychology (pp. 559-601). New Jersey: Pretice-Hall.

Encuesta Nacional de Salud (2003). Madrid: Instituto Nacional de Estadística. Recuperado el 9 de Febrero de 2010, de http://www.ine.es/inebase/cgi.

García, J.L., Avis, M., Cobos, F., Biurrum, A.C., Eslava, J.L., Rodrigo, C., Padilla, B., y Tinajas, M.R. (1995). Conductas sexuales de riesgos en universitarios-as: Un estudio en la Universidad Pública de Navarra. Cuadernos de Medicina Psicosomática y Psiquiatría de Enlace, (36), 48-60.

Gascón, J.A., Navarro, B., Gascón, F.J., Férula, L.A., Jurado, A., y Montes, G. (2003). Comportamiento sexual de los escolares adolescentes en la ciudad de Córdoba. Atención Primaria, (32), 355-60.

Gras, M.E., Planes, M., Soto, J., y Font-Mayolas, S. (2000). Percepción de riesgo y comportamientos heterosexuales relacionados con el SIDA: estudio comparativo con cinco muestras de universitarios. Cuadernos de Medicina Psicosomática y Psiquiatría de Enlace, (54), 39-45.

Hidalgo, I., Garrido, G., y Hernández, M. (2000). Health status and risk behaviour of adolescents in the north of Madrid. Spain. Journal of Adolescent Health, (27), 31-360.

INE (2003). Encuesta de Salud y Hábitos Sexuales. Madrid: Instituto Nacional de Estadística.

Injuve, (1996). Informe Juventud en España (1996). Madrid: Ministerio de Trabajo y Asuntos Sociales.

Injuve, (2000). Informe de la Juventud en España 2000. Madrid: Ministerio de Trabajo y Asuntos Sociales.

Injuve, (2004). Sondeo de opinión y situación de la gente joven. Segunda encuesta de 2004. Madrid: Ministerio de Trabajo y Asuntos Sociales.

Injuve, (2008). Informe Juventud en España (2008). Madrid: Ministerio de Trabajo y Asuntos Sociales.

Lameiras, M. y Failde, J.M. (1997). Sexualidad y salud en jóvenes universitarios: actitud, actividad sexual y percepción de riesgo de la transmisión heterosexual del VIH. Análisis y Modificación de Conducta, 23 (93), 28-63.
Lameiras, M., Castro, Y., Calado, M., y González, M. (2004b). Determinantes del inicio de las relaciones sexuales en adolescentes españoles. Cuadernos de Medicina Psicosomática y Psiquiatría de Enlace, (7172), 67-75.

Lasheras, M.G., Cuñe, J., Bautista J., y Farré J.M. (2005). Hábitos sexuales en jóvenes universitarios. Cuadernos de Medicina Psicosomática y Psiquiatría de Enlace, (74), 57-63.

Mesa, M.I., Barella, J.L., y Cobeña, M. (2004). Comportamientos sexuales y uso de preservativos en adolescentes de nuestro entorno. Atención Primaria, 33 (7), 374-380

Oraá, R. (1996). Hábitos sexuales y conducta preventiva de los/as jóvenes de la Rioja. Cuadernos de Medicina Psicosomática y Psquiatria de Enlace, (40), 51-61.

Ramírez, Y., García, J., De las casas, I., García, E., y Godines, J.A. (2002). Encuesta sobre uso de métodos anticonceptivos en Andalucía. Revista Iberoamericana de Fertilidad y Reproducción Humanas, (VI Congreso SEC), 138-139.

Rodríguez, J. y Traverso, C.I. (2007). Conductas y Educación sexual en universitarios españoles. Málaga: Grupo Editorial.

Segunda Encuesta Schering Sexualidad y Anticoncepción en la Juventud Española (2005). Recuperado el 23 de Enero de 2008, de http://www.equipodaphane.es.

Sueiro, E. y Diéguez J.L. (2001). Juventud y sexualidad (pp. 257-269). Actas do VI Congreso GalaicoPortuguês de Psicopedagogía. Braga

Teva, I., Bermúdez, M.P., y Buela-Casal, G. (2009b). Conductas de riesgo para la infección por el VIH y las enfermedades de transmisión sexual (ETS) en adolescentes en el año 2007: diferencias en función de variables sociodemográficas. Revista Española de Salud Pública, (83), 309-320. 